\title{
Update on Liver Transplantation: What is New Recently?
}

\author{
Burcak E Tasdogan ${ }^{1}$, Sinan Akosman ${ }^{2}$, Merve Gurakar ${ }^{3}$, Cem Simsek $^{4}$, Ahmet Gurakar ${ }^{5}$
}

\begin{abstract}
Liver transplantation (LT) is an evolving area of medicine for the treatment of certain types of malignancies and acute and chronic liver failures. Since the topic is evolving, new literature is increasingly available. In recent years, with the emerging potent antiviral therapies, hepatitis $C$ virusinfected patients have successful patient and graft survival outcomes. Even human immunodeficiency virus (HIV) positive patients previously contraindicated for organ transplantation are transplanted with comparable outcomes. With increasing demand for LT, the shortage of the donor pool became the rate limiting factor in this hopeful treatment. To overcome waitlist mortality and expand the donor pool, scoring systems have been modified and organs from HIV and/or hepatitis $\mathrm{C}$ infected donors are now accepted, under certain circumstances. The new literature also questions the 6-month alcohol abstinence rule for the transplantation of alcoholic liver disease (ALD), in light of early transplantation results from severe alcoholic hepatitis (SAH) trials.
\end{abstract}

Keywords: Direct-acting antiviral drugs, Donor pool, Highly active antiretroviral therapy, Liver transplantation, Scoring system, Severe alcoholic hepatitis.

Euroasian Journal of Hepato-Gastroenterology (2019): 10.5005/jp-journals-10018-1293

\section{INTRODUCTION}

$\mathrm{O}$ gan transplantation is an incredible area of medicine which cures patients with medically incurable disease. Clinicians' desperation for certain types of malignancies and acute and chronic organ failures accelerated the evolution of organ transplantation worldwide. Since the topic is evolving, new literature is an increasingly available and challenging follow-up. In this review, we evaluate recent literature related to LT.

\section{Scoring Systems and Effect of Serum Sodium Levels on Outcome}

In organ transplant, organ allocation is one of the first and most important steps in successful transplantation. The systems in place for organ allocation and distribution are never perfect, but certain calculations can facilitate the process. For many years, clinicians sought to optimize transplant candidacy selection and timing. Timing is important because patients should undergo surgery before life-threatening systemic complications of the disease occur.

On the other hand, patients should not be transplanted too early, since the long-standing immunosuppression therapy required after surgery may cause complications. As demand for transplantation increased, the limited availability of cadaveric organs became the main limiting factor. Waiting lists grew longer, and increased mortality among patients awaiting transplantation became the issue. In response, national organ allocation systems initiated scoring systems based on disease severity and complications to give priority to specialized patients.

The first scoring system, Child-Turcotte-Pugh, was used in 1997 to asses disease severity. Since it relied on subjective data about hepatic encephalopathy and ascites, however, inequities were perceived. In 2002, the model for end-stage liver disease (MELD) model was introduced, which is a logarithmic score based on objective parameters. It is valuable in determining the 3-month mortality of LT candidates without transplantation. Transplanted patients with MELD $\leq 14$ had a shorter 1-year survival versus MELD $\leq 14$ patients without transplantation. One year after the introduction of the MELD score there was a $12 \%$ reduction in the number of new candidates due to low MELD score and a 3.4\% reduction at 5 years. ${ }^{1-3}$ MELD decreased the median time to transplantation, with a higher
1,4,5The Johns Hopkins University School of Medicine, Division of Gastroenterology and Hepatology, Baltimore, Maryland, United States

${ }^{2}$ The Johns Hopkins University, Baltimore, Maryland, United States

${ }^{3}$ The Johns Hopkins University, Bloomberg School of Public Health, Baltimore, Maryland, United States

Corresponding Author: Ahmet Gurakar, The Johns Hopkins University School of Medicine, Division of Gastroenterology and Hepatology, Baltimore, Maryland, United States, Phone: +1 410614 3369, e-mail: aguraka1@jhmi.edu

How to cite this article: Tasdogan BE, Akosman S, et al. Update on Liver Transplantation: What is New Recently? Euroasian J Hepatogastroenterol 2019;9(1):34-39.

Source of support: Nil

Conflict of interest: None

proportion of patients undergoing transplantation within 30 days of listing. ${ }^{3}$ Despite these advantages, some weaknesses of the MELD score were also reported. The MELD score excessively increases by other causes of hyperbilirubinemia and renal diseases, and thus provides an advantage to patients who have these conditions for transplantation. A second disadvantage is that it excludes the clinical judgment of the providers and disability or quality of life of the patients. Therefore, for these clinical situations and malignancies extra points were given (Table 1).

However, the extra points are given to hepatocellular carcinoma (HCC) and the automatic increases over time led to debate amongst clinicians. Most HCCs are treated with interventional locoregional therapies, like transarterial chemoembolization or radiofrequency ablation at the time of diagnosis. These patients can stay in remission for long periods, and their survival may be longer than non-HCC patients. It was determined that extra points for HCC were unfair to the waiting patients on the transplant list, by way of the higher score will yield higher rates of transplantation, shorter waiting times and lower mortality during the waitlist period. ${ }^{4}$ In 2009 , a different system for exception points, which the diameter and number of HCC lesions were taken into consideration, was suggested as Québec MELD HCC exception point system. Every 3 months imaging was repeated to reassess tumor burden and investigate

(c) The Author(s). 2019 Open Access This article is distributed under the terms of the Creative Commons Attribution 4.0 International License (https://creativecommons. org/licenses/by-nc/4.0/), which permits unrestricted use, distribution, and non-commercial reproduction in any medium, provided you give appropriate credit to the original author(s) and the source, provide a link to the Creative Commons license, and indicate if changes were made. The Creative Commons Public Domain Dedication waiver (http://creativecommons.org/publicdomain/zero/1.0/) applies to the data made available in this article, unless otherwise stated. 
Table 1: Clinical situations in which extra points are granted

\begin{tabular}{l}
\hline Refractory ascites \\
\hline Recurrent gastrointestinal bleeding \\
Recurrent or chronic hepatic encephalopathy \\
Hepatopulmonary syndrome \\
Portopulmonary hypertension \\
Intractable pruritus resistant to medical therapies \\
Budd-Chiari syndrome \\
Familial amyloidotic polyneuropathy \\
Cystic fibrosis \\
Hereditary hemorrhagic telengiectasia \\
Polycystic liver disease \\
Primary hyperoxaluria \\
Recurrent cholangitis \\
Uncommon metabolic diseases \\
Malignancies (cholangiocarcinoma, hepatocellular carcinoma, \\
uncommon liver tumors)
\end{tabular}

tumor growth. This scoring system did not significantly shorten waitlist times for HCC patients compared to the cohort before 2009. However, it did not have a positive effect on pretransplant mortality posttransplant graft and patient survivals for 1 and 2 years. ${ }^{5}$ The analysis showed no positive effect on outcomes, so the Québec MELD HCC exception point system was not accepted by the general community or used by national allocation programs.

Of the clinical situations, influencing the quality of life, ascites and dilutional hyponatremia are most commonly investigated. Both ascites and hyponatremia were shown to predict the waitlist mortality independent of their MELD score, especially when the MELD score is less than 216. In January 2016, serum sodium level was added to the MELD score formulation for any patient with a MELD over 11 to make a more accurate prediction of pretransplant mortality. This formula (MELD-Na) is calculated as MELD $+1.32 \times$ (137-Na)-[0.033 $\times$ MELD*(137-Na)]. In addition to waitlist mortality, sodium level is also implicated in post-LT mortality. Many studies were conducted to study outcomes in patients with hyponatremia. Two European studies showed shortened post-LT survival among patients with pretransplant hyponatremia., ${ }^{7,8}$ The larger study consisting of 5,152 liver recipients showed that lower than 130 serum $130 \mathrm{mEq} / \mathrm{L}$ sodium levels were associated with a significantly higher in 90-day posttransplant mortality. ${ }^{8}$ Another study of 19,537 patients found a conflicting result concluding that patients with different measures of pre-LT hyponatremia, in fact, have similar post-LT 90-day survivals compared to normonatremic recipients. ${ }^{9}$ Two smaller studies have validated this result. ${ }^{10,11}$ Opposite findings in European trials have been attributed to differentrecipient and donor characteristics, as well as different organ allocation systems based onwaiting time rather than disease severity. Fragile patients with higher MELD scores and hyponatremia wait for a longer time period allowing complications to develop and affect posttransplantation outcomes. On the other hand, hypernatremia was found to significantly decrease post-LT survival. The negative effect of hypernatremia on post-LT survival held constant even after adjustment for greater moderate to severe ascites, grade 3-4 hepatic encephalopathy, and higher MELD scores in hypo- and hypernatremic groups versus normonatremic patients. ${ }^{9}$

\section{Hepatitis C Virus Infection}

There are many reasons for liver failure and a need for LT. The leading reason in the United States and numerous other European countries is chronic hepatitis $\mathrm{C}(\mathrm{HCV})$. A retrospective study showed that, among the 74,216 recipients from 2002 to 2015, the leading etiology for LT was HCV $(22.4 \%, n=16,627) .{ }^{12}$ Immunosuppressive drugs given after transplantation affect the cellular immune system, accelerating the course of viral infections including HCV. Since interferon-based therapies were hard to tolerate and had low response rates, post-LT $\mathrm{HCV}$ infection with more rapid fibrosis and liver dysfunction was a major clinical concern. In a retrospective cohort study comparing the outcomes of $L T$ in $\mathrm{HCV}$ positive patients versus patients transplanted for other indications. Former group showed higher graft failure and mortality rates. ${ }^{13}$ Post-LT HCV infection led to accelerated liver injury causing cirrhosis with faster decompensation in a short period of time. Once decompensation occurs, 3-year survival is $<10 \%$ which is $>60 \%$ for an immunocompetent decompensated HCV related cirrhosis patient. ${ }^{14}$ Worst of all, in $10 \%$ of recipients, HCV recurrence is accompanied by an aggressive entity called fibrosing cholestatic hepatitis (FCH). The laboratory work often shows a high viral load with the evolution of divergent quasispecies and there is significant liver dysfunction eventually leading to $90 \%$ mortality.

For the last 5 years, $\mathrm{HCV}$ is no longer a concern. Highly effective direct-acting antiviral drugs (DAA) cure the infection with a high sustained virologic response (SVR) rate and minimize the injury. The timing of DAA therapy is a discussion point. When the drugs are administered before LT, patients' MELD scores decrease without an improved quality of life, resulting in a disadvantage on the waitlist. As per the 2016 EASL guidelines, patients with higher than 18 MELD score must have therapy postponed to the post-transplant period. The treatment continues for 3-6 months depending on the genotype and/or ribavirin consumption, which may be too late for a fragile and complicated patient.

Additionally, HCV-positive patients have the opportunity to take organs from the HCV-positive donor pool. By treating them, they lose this chance which especially disadvantageous in areas where the HCV-positive donor pool is large. A study done in our center investigated hepatitis C infected recipients from 2014 to 2015 according to their HCV treatment period. Among the 67 patients, 21 of them were treated pre-LT. Twenty-five of 46 who were HCVpositive at the time of surgery received HCV-positive livers. The waiting time was shorter for the untreated group (287 versus 172 days). ${ }^{15}$ These points highlight the advantages of post-LT therapy. Another consequential risk factor for virus recrudescence is the duration of viral load negativity in the pretransplant period which is inversely proportional to recurrence. If viral load is negative for over 30 days before the LT, the likelihood of recrudescence is $<95 \%$. ${ }^{16}$

Since subjects are prone to renal injury and anemia from immunosuppressive regimens, the addition of ribavirin into antiviral therapy is also controversial. In a ribavirin-free trial consisting of 62 post-LT patients [34 cirrhosis (55\%), 1 FSC (2\%)], subjects were treated with second-generation DAA combinations and followed up for 96 weeks after the cessation of the treatment. Six patients died during the trial with multiorgan failure in four of them. With the intention to treat analysis, sustained viral response (SVR) at the 96th week was $89 \%(n=55 / 62) .{ }^{17}$ Another trial enrolled 87 post-LT patients (81.6\% cirrhosis) who were given 24 -week DAA \pm ribavirin. The ribavirin-treated group comprised $59.8 \%(n=52)$. Five cirrhotic patients, four of them in the ribavirin-free group, died. ${ }^{12}$ Week SVR was $80 \%$ in the ribavirin-free and $98.1 \%$ in the ribavirin-treated groups ( $p>0.05) .{ }^{18}$ More successful results are found in noncirrhotic patients. Sofosbuvir + ribavirin combination was administered for 24 weeks to 63 noncirrhotic posttransplant patients and 12 and 
24-week SVRs after the cessation of the treatment was $95.2 \%(n=60)$ and $93.7 \%(n=59)$, respectively. Factors related to relapse were noted as long treatment-free duration after the transplantation, older age, higher fibrosis score and erythropoietin need during the treatment. ${ }^{19}$ In conclusion, ribavirin can be used safely in a specific population, giving them a chance for a shorter treatment duration without any difference in the outcome.

$\mathrm{HCV}$ recurrence is more rapid in patients who received a liver from donors after cardiac death (DCD) versus donors after brainstem death. Two groups of 44 post-LT patients with matched features were enrolled and followed. Time for HCV recurrence after transplantation was significantly shorter in the DCD group (408 vs. 567 days), so it is advised that early post-LT antiviral therapy should be given to DCD recipients to prevent graft injury. ${ }^{20}$

Given that longer duration of pretransplant HCV viral clearance, shorter time to HCV treatment post-LT, and lower fibrotic degree of the liver favorably affect post-transplant outcomes, we suggested a new therapy choice called "bridging therapy". Four HCV positive live donor recipients were treated with at least four weeks of genotypespecific DAA according to the AASLD guideline, and transplantation was scheduled after viral load negativity was achieved. One month of Sofosbuvir maintenance treatment was given to the recipients just after the surgery. One patient died due to multiorgan failure at 4 months after transplantation, no serum sample was obtained. The other three patients achieved SVR 12 weeks after the cessation of the therapy. ${ }^{21}$ With these results, "bridging therapy" seems to be a successful therapy option for live donor transplanted patients and must be studied in larger cohorts.
With the rising demand for solid organ transplantation and diminished donor pool, waitlist mortality and dropouts due to severe disease are increasing. It is prudent to use all available resources to expand the donor organ supply, and our data support the practice of using extended-criteria DCD grafts. Drug addiction is expanding worldwide with increased mortality as a result of drug overdose. These expired drug users' organs may be donated, but are usually discarded for being HCV and/or HIV positive. These organs have the potential to save lives because they are usually coming from young donors who are otherwise healthy. Utilization of HCV-positive organs for HCV-positive recipients has been carried out by some transplantation centers and the outcomes are comparable with those who receive HCV-negative allografts. ${ }^{22}$ The American Society of Transplantation recently encouraged clinicians to form prospective research protocols that compare the risks and benefits of using HCV- positive organs. ${ }^{23}$ This new opportunity to widen the donor pool with HCV positive grafts is due to DAA usage. Their effect is so reliable that in some transplantation units, HCV negative patients with serious liver disease complications who have no time to wait for a donation are offered hepatitis $C$ positive organs which they accept with favorable outcomes with early post-LT DAA therapy. ${ }^{24}$ Of note, some DAA may interact with the immunosuppressive drugs and result in altered serum drug levels (Table 2).

\section{Human Immunodeficiency Virus Infection}

Improvements in highly active antiretroviral therapy (HAART) led to an increased life expectancy for HIV positive patients.

Table 2: Possible drug interactions between DAAs and immunosuppressive drugs

\begin{tabular}{|c|c|c|c|c|c|c|c|c|c|c|}
\hline & $\begin{array}{l}\text { Elbasvir/ } \\
\text { grazoprevir }\end{array}$ & $\begin{array}{l}\text { Ombitasvir/ } \\
\text { paritaprevir/ } \\
\text { ribavirin }\end{array}$ & $\begin{array}{l}\text { Ombitasvir/ } \\
\text { paritaprevir/ } \\
\text { ribavirin/ } \\
\text { dasabuvir }\end{array}$ & Simeprevir & $\begin{array}{l}\text { Sofosbuvir/ } \\
\text { velpatasvir/ } \\
\text { voxilaprevir }\end{array}$ & Daclatasvir & $\begin{array}{l}\text { Glecaprevir/ } \\
\text { pibrentasvir }\end{array}$ & $\begin{array}{l}\text { Ledipasvir/ } \\
\text { sofosbuvir }\end{array}$ & $\begin{array}{l}\text { Sofosbuvir/ } \\
\text { velpatasvir }\end{array}$ & Sofosbuvir \\
\hline CSA & $\begin{array}{l}\text { Increase } \\
\text { grazoprevir } \\
\text { AUC by } 15- \\
\text { fold }\end{array}$ & $\begin{array}{l}\text { Increase } \\
\text { CSA AUC } \\
\text { by 4-fold, } \\
\text { increase } \\
\text { paritaprevir } \\
46 \%\end{array}$ & $\begin{array}{l}\text { Increase CSA } \\
\text { AUC by 5.8- } \\
\text { fold }\end{array}$ & $\begin{array}{l}\text { Increase } \\
\text { simeprevir } \\
\text { AUC by } \\
5.81 \text {-fold }\end{array}$ & $\begin{array}{l}\text { Concentration } \\
\text { of voxilaprevir } \\
\text { increase by } \\
\text { 19-fold }\end{array}$ & $(-)$ & $\begin{array}{l}\text { Not } \\
\text { recommended } \\
\text { for patients } \\
\text { requiring CSA } \\
\text { doses }>100 \\
\text { mg/day }\end{array}$ & $(-)$ & $(-)$ & $(-)$ \\
\hline TAC & $\begin{array}{l}\text { Increase TAC } \\
\text { AUC by } 43 \%\end{array}$ & $\begin{array}{l}\text { Increase } \\
\text { TAC AUC by } \\
86 \text {-fold }\end{array}$ & $\begin{array}{l}\text { Increase TAC } \\
\text { AUC by 57- } \\
\text { fold }\end{array}$ & $\begin{array}{l}\text { Increase } \\
\text { simeprevir } \\
\text { AUC by } 85 \% \text {, }\end{array}$ & & & & & & \\
\hline $\begin{array}{l}\text { Decrease } \\
\text { TAC AUC } \\
\text { by } 17 \%\end{array}$ & $\begin{array}{l}\text { Increase } \\
\text { TAC AUC by } \\
9 \% \text {, increase } \\
\text { sofosbuvir } \\
\text { AUC by } 13 \%\end{array}$ & $(-)$ & $\begin{array}{l}\text { Increase TAC } \\
\text { AUC by } 1.45- \\
\text { fold }\end{array}$ & $(-)$ & $(-)$ & $(-)$ & & & & \\
\hline$A Z A$ & $(-)$ & $(-)$ & $(-)$ & $(-)$ & $(-)$ & $(-)$ & $(-)$ & $(-)$ & $(-)$ & $(-)$ \\
\hline MMF & $(-)$ & Unknown & Unknown & $(-)$ & $(-)$ & $(-)$ & $(-)$ & $(-)$ & $(-)$ & $(-)$ \\
\hline SRL & Unknown & $\begin{array}{l}\text { Increase } \\
\text { SRL AUC by } \\
\text { 38-fold }\end{array}$ & $\begin{array}{l}\text { Increase SRL } \\
\text { AUC by 38- } \\
\text { fold }\end{array}$ & Unknown & Unknown & $(-)$ & Unknown & $(-)$ & $(-)$ & $(-)$ \\
\hline EVR & Unknown & $\begin{array}{l}\text { Increase EVR } \\
\text { AUC by } 27.1- \\
\text { fold }\end{array}$ & $\begin{array}{l}\text { Increase EVR } \\
\text { AUC by 27.1- } \\
\text { fold }\end{array}$ & Unknown & Unknown & $\begin{array}{l}\text { May } \\
\text { increase } \\
\text { EVR concen } \\
\text { tration }\end{array}$ & Unknown & $\begin{array}{l}\text { May } \\
\text { increase } \\
\text { EVR } \\
\text { concen } \\
\text { tration }\end{array}$ & Unknown & $(-)$ \\
\hline
\end{tabular}

CSA, cyclosporine A; TAC, tacrolimus; AZA, azathioprine; MMF, mycophenolate mofetyl; SRL, sirolimus; EVR, everolimus; AUC, area under the plasma drug concentration-time curve (reflects the actual body exposure to drug after administration of a dose of the drug) (https://www. hep-druginteractions.org) 
Opportunistic infections are no longer the leading cause of death in well-treated HIV positive patients. With increased survival, other systemic illnesses like hypertension, diabetes mellitus, and hyperlipidemia normally seen in older adults and which cause predisposition to nonalcoholic steatohepatitis and nephropathy are frequent. Also, coinfections like HCV and hepatitis B, alcohol and drug addictions aggravate kidney and liver damage and HIV itself, may cause nephropathy and hepatopathy/cholangiopathy. From 1999 to 2010, HIV (+) end-stage kidney disease increased 14-fold in number. ${ }^{25}$ For these reasons, end-stage organ diseases requiring consideration for organ transplantation recently became frequent in this population.

Solid organ transplantation was historically a contraindication for HIV-infected patients. Prior transplantation experience in HIV positive patients included who were not diagnosed before or who were infected after solid organ transplantation. Recipients had not received appropriate and effective antiretroviral therapy and were missing crucial pretransplantation data such as CD4 lymphocytes count and viral load important to determining the infection's long-term prognosis. With posttransplant immunosuppression treatment, an accelerated course of HIV infection occurred and, transplantation outcomes were worse than for noninfected patients. ${ }^{26,27}$ HAART was introduced in 1996 improving the HIV related outcomes of patients and giving them the opportunity for transplantation. An analysis of 249 HIV (+) liver recipients, 55\% also HCV (+), transplanted between 2002 and 2012 with 2.4 years of median follow-up had 1 and 3-year graft survivals of $74 \%$ and $59 \%$, respectively. ${ }^{28}$ Another study involving 11 stable HIV (+) liver recipients with a median 3.4 year follow-up showed 1-year patient and graft survivals $91 \%$ and $64 \%$, respectively. Longer follow-up yielded 3-year patient and graft survivals of $82 \%$ and $64 \%$ respectively. In this trial, all four recipients expired due to HCV related liver disease complications and liver failure. ${ }^{29}$ These rates are comparable with the older HIV (-) liver recipient rates. At the time those trials were conducted, historic interferon-based therapy was given for HCV infection which may explain the lower survival rates compared with the age group. We assume that with the new DAA therapy the outcomes would be much better, becoming comparable with age-matched HIV-uninfected ones. To determine the effect of DAA drugs on HIV coinfected patients, studies were conducted which showed hopeful results. HCV-HIV coinfected 29 post-LT patients were treated with sofosbuvir based DAA therapy, 6 of them were FCH. SVR rate at 12 weeks was $96.6 \%{ }^{30}$ The drug-drug interactions may be confusing for clinicians since immunosuppressive, HAART and DAA agents are given together in these patients. Immunosuppressive drug levels in the post-LT period must be followed early and frequently with stable anti-viral therapy.

HIV positive liver recipients are found to have longer waitlist times according to MELD and increased waitlist mortality compared to HIV negative ones. But in these trials, some of the subjects involved had uncontrolled HIV infection with lower CD4 cell counts and positive viral load. With this result, authors made a comment to calculate a different score for HIV (+) patients for a better predictor of survival, like MELD CD4 score. ${ }^{31,32}$ However, in a latter trial surveying the waitlists for LT from 2003 to 2007, 58 out of 167 HIV (+) patients (34.7\%) and 377 out of 792 matched HIV (-) patients (47.6\%) were transplanted and the pretransplant waitlist mortalities were similar between the two groups. Further analysis showed that the MELD score $(p<0.0001)$ was the only factor influencing waitlist mortality. CD4 (+) cell count and viral load had no effect. ${ }^{33}$ This clears the fact that for treatment controlled HIV-infected patients, waitlist mortality is similar to HIV (-)
ones.MELD is a reliable predictor of pretransplant mortality in both groups so the incorporation of MELD into the management of HIV positive patients should be encouraged.

The number of HIV-infected donors is increasing like the number of HIV-infected end-stage chronic organ failure patients. In Cape Town and Johannesburg, the seroprevalence of HIV in potential donors referred to transplant coordinators was determined as $10-20 \% .{ }^{34}$ Since the numbers are increasing for both donors and recipients, the expansion of the donor pool might be a good approach. With the new treatment strategies for HIV and HCV, comparable survival results and increasing HIV positive patients' organ demand and HIV positive donor pool, the HIV (+) to HIV (+) organ transplantation has been legal in USA since 2013, under a research protocol named "HIV Organ Policy Equity" (HOPE) Act. ${ }^{25}$ First HOPE Act Liver Transplantation was performed at John Hopkins Hospital on March 19, 2016. Nevertheless, organ transplantation in HIV positive population is a relatively new area. We still need more studies regarding the appropriate selection of recipients and donors, post-transplant medical management strategies and characteristics of organ rejection in this population.

\section{Transplantation for Acute Alcoholic Hepatitis}

ALD is a clinic spectrum of conditions where the liver has different degrees of injury according to predisposing factors. Hepatosteatosis, acute alcoholic hepatitis, and cirrhosis are in this clinical spectrum. ALD follows HCV and HCC as a third leading indication for LT after of the 74,216 performed LTs in the USA from 2002 to $2015 .^{12}$ Transplantation centers usually require a 6-month sobriety period for ALD before surgery, but this time period may be too long for severe alcoholic hepatitis (SAH) patients who are characterized with rapid onset of jaundice and Maddrey's discriminant function $>32 .{ }^{35,36}$ The medical management of SAH is corticosteroid therapy with supportive measures. After one week of high dose corticosteroid therapy, Lille score is calculated which predicts the benefit of the treatment. If it is $>0.45$ at the end of one week of therapy, it means that the patient would get no benefit from further treatment. ${ }^{37}$ The other predictive value is a MELD score. The continuous increase in MELD score despite therapy is also consistent with poor prognosis. There is no other treatment option proven to be effective in these steroid-resistant patients and the reported 1 and 6-month mortality is above $50 \%$ and $70 \%$ in treatment failure patients, respectively. ${ }^{38-41}$ Also, these patients are usually young and otherwise healthy individuals, so 6-month sobriety requirement free $L T$, which is also called early $L T$, must be considered under these circumstances. This topic is a fresh area of $L T$ with few publications. In trials, patients considered for early transplantation were severe, steroid nonresponder subgroup of alcoholic hepatitis with strong social support and commitment to abstinence. The first trial was a case-control study from Europe examining the outcomes of 26 SAH patients with early transplantation. Transplanted patients had a significantly longer rate of 6 -month survival rate (77\%) compared to matched medically managed control group (23\%) $(p<0.001)$. Recidivism is reported as $11.5 \%(n=3)$ similar to the rate of alcoholic cirrhosis cases with 6-month sobriety, stated in the previous analysis. ${ }^{42,43}$ The outcomes of alcoholic liver disease indicated transplantations from our center was published recently. From 2012-2017, 34 alcoholic cirrhosis patients with 6 months of alcohol abstinence and $46 \mathrm{SAH}$ patients were given liver transplant. After a median follow-up of 532 days (281-998 days), there were no differences between two groups regarding 6-month and 1-year graft and patient survivals. The recidivism rate was similar, too, $24 \%$ for cirrhosis patients and $28 \%$ for SAH ( $p=0.8)$. For patients with 6 
months of pretransplant abstinence, a longer duration of abstinence did not lower the risk of recidivism.(HR 1.00; $95 \% \mathrm{Cl} 0.998$ to 1.001 ; $p=0.6)$. Further analysis showed that recipients' younger than 50 age and higher Hopkins psychosocial scale (a scoring model identifying the risk of alcoholism relapse) score and presence of SAH predicted alcohol relapse. No risk factors for cirrhosis patients were shown. ${ }^{44}$ Since SAH patients' outcomes are not different from alcoholic cirrhosis patients' outcomes on waitlists, early LT might be a good option for a selected group of SAH patients who have predictors of high mortality.

\section{Conclusion}

LT is an evolving part of medicine which gives patients a "second chance" by resolving life-threatening acute or chronic liver diseases. In recent years, relying on strong evidence, early LT without a 6-month alcohol abstinence rule is suggested for selected patients with $\mathrm{SAH}$. With emerging immunosuppressive drugs and antiviral therapies, the posttransplant patient and graft outcomes are successful, but the shortage of the donor pool is the speed limiting part of this hopeful treatment. To overcome this slowdown, organ allocation scoring systems have changed to decrease waitlist mortality. They select the"sickest" patient first for whom the transplantation would be most beneficial and appropriate timing wise. To widen donor pools, transplantation of HIV positive organs is permitted under the HOPE Act since 2013. Similarly, HCV positive to positive and even positive to negative organ transplantation is safely carried out under a research protocol in some centers using the highly effective DAA to cure the posttransplant HCV infection.

\section{References}

1. Merion RM, Schaubel DE, et al. The survival benefit of liver transplantation. American journal of transplantation: official journal of the American Society of Transplantation and the American Society of Transplant Surgeons 2005;5(2):307-313.

2. Freeman RB, Wiesner $\mathrm{RH}$, et al. Results of the first year of the new liver allocation plan. Liver Transpl 2004;10(1):7-15.

3. Thuluvath PJ, Guidinger MK, et al. Liver transplantation in the United States, 1999-2008. American journal of transplantation: official journal of the American Society of Transplantation and the American Society of Transplant Surgeons 2010;10(4 Pt 2):1003-1019.

4. Northup PG, Intagliata NM, et al. Excess mortality on the liver transplant waiting list: unintended policy consequences and Model for End-Stage Liver Disease (MELD) inflation. Hepatology 2015;61(1):285-291.

5. Bhat $M$, Ghali $P$, et al. Proposal of a novel MELD exception point system for hepatocellular carcinoma based on tumor characteristics and dynamics. J Hepatol 2017;66(2):374-381.

6. Heuman DM, Abou-Assi SG, et al. Persistent ascites and low serum sodium identify patients with cirrhosis and low MELD scores who are at high risk for early death. Hepatology 2004;40(4):802-810.

7. Londono MC, Guevara M, et al. Hyponatremia impairs early posttransplantation outcome in patients with cirrhosis undergoing liver transplantation. Gastroenterology 2006;130(4):1135-1143.

8. Dawwas MF, Lewsey JD, et al. The impact of serum sodium concentration on mortality after liver transplantation: a cohort multicenter study. Liver Transpl 2007;13(8):1115-1124.

9. Leise $M D$, Yun BC, et al. Effect of the pretransplant serum sodium concentration on outcomes following liver transplantation. Liver Transpl 2014;20(6):687-697.

10. Yun BC, Kim WR, et al. Impact of pretransplant hyponatremia on outcome following liver transplantation. Hepatology 2009;49(5):16101615.

11. Hackworth WA, Heuman DM, et al. Effect of hyponatraemia on outcomes following orthotopic liver transplantation. Liver Int 2009;29(7):1071-1077.
12. Kling CE, Perkins JD, et al. Recent trends in liver transplantation for alcoholic liver disease in the United States. World J Hepatol 2017;9(36):1315-1321.

13. Forman LM, Lewis JD, et al. The association between hepatitis $\mathrm{C}$ infection and survival after orthotopic liver transplantation. Gastroenterology 2002;122(4):889-896.

14. Gane EJ. The natural history of recurrent hepatitis $C$ and what influences this. Liver Transpl 2008;14 Suppl 2:S36-544.

15. Abdelqader A, Kabacam G, et al. Pre- Versus Posttransplant Treatment of Hepatitis C Virus With Direct-Acting Antivirals in Liver Transplant Recipients: More Issues to be Solved. Exp Clin Transplant 2017;15(Suppl 1):1-5.

16. Curry MP, O'Leary JG, et al. Sofosbuvir and Velpatasvir for HCV in Patients with Decompensated Cirrhosis. N Engl J Med 2015;373(27):2618-2628.

17. Beinhardt $\mathrm{S}$, Al-Zoairy $\mathrm{R}$, et al. Long-term follow-up of ribavirin-free DAA-based treatment in HCV recurrence after orthotopic liver transplantation. Liver Int 2017.

18. Lionetti R, Calvaruso V, et al. Sofosbuvir plus daclatasvir with or without ribavirin is safe and effective for post-transplant hepatitis $C$ recurrence and severe fibrosis and cirrhosis: A prospective study. Clin Transplant 2018;32(2).

19. Anand AC, Agarwal SK, et al. Sofosbuvir and Ribavirin for 24 Weeks Is An Effective Treatment Option for Recurrent Hepatitis C Infection After Living Donor Liver Transplantation. J Clin Exp Hepatol 2017;7(3):165-171.

20. Townsend SA, Monga MA, et al. Hepatitis C Virus Recurrence Occurs Earlier in Patients Receiving Donation After Circulatory Death Liver Transplant Grafts Compared With Those Receiving Donation After Brainstem Death Grafts. Transplant Proc 2017;49(9):2129-2134.

21. Niranjan-Azadi AM, Kabacam G, et al. Single-Center Experience in Pretransplant Hepatitis C Virus (HCV) Treatment Among Living Donor Liver Transplant Candidates: Bridging the Direct-Acting Antivirals (DAA). Ann Transplant 2017;22:570-574.

22. Northup PG, Argo $C K$, et al. Liver allografts from hepatitis $C$ positive donors can offer good outcomes in hepatitis $C$ positive recipients: a US National Transplant Registry analysis. Transpl Int 2010;23(10):10381044.

23. Levitsky J, Formica RN, et al. The American Society of Transplantation Consensus Conference on the Use of Hepatitis C Viremic Donors in Solid Organ Transplantation. American journal of transplantation: official journal of the American Society of Transplantation and the American Society of Transplant Surgeons 2017;17(11):2790-2802.

24. Saberi B, Hamilton JP, et al. Utilization of hepatitis C virus RNA-positive donor liver for transplant to hepatitis C virus RNA-negative recipient. Liver Transpl 2018;24(1):140-143.

25. Boyarsky BJ, Durand CM, et al. Challenges and Clinical DecisionMaking in HIV-to-HIV Transplantation: Insights From the HIV Literature. American journal of transplantation: official journal of the American Society of Transplantation and the American Society of Transplant Surgeons 2015;15(8):2023-2030.

26. Gow PJ, Pillay D, et al. Solid organ transplantation in patients with HIV infection. Transplantation 2001;72(2):177-181.

27. Roland ME, Stock PG. Review of solid-organ transplantation in HIVinfected patients. Transplantation 2003;75(4):425-429.

28. Campos-Varela I, Dodge JL, et al. Key donor factors associated with graft loss among liver transplant recipients with human immunodeficiency virus. Clin Transplant 2016;30(9):1140-1145.

29. Roland ME, Barin B, et al. HIV-infected liver and kidney transplant recipients: 1-and 3-year outcomes. American journal of transplantation: official journal of the American Society of Transplantation and the American Society of Transplant Surgeons 2008;8(2): 355-365.

30. Antonini TM, Coilly A, et al. Sofosbuvir-Based Regimens in HIV/HCV Coinfected Patients After Liver Transplantation: Results From the ANRS CO23 CUPILT Study. Transplantation 2018;102(1):119-126.

31. Ragni MV, Eghtesad B, et al. Pretransplant survival is shorter in HIVpositive than HIV-negative subjects with end-stage liver disease. Liver Transpl 2005;11(11):14251430. 
32. Pineda JA, Romero-Gomez M, et al. HIV coinfection shortens the survival of patients with hepatitis $C$ virus-related decompensated cirrhosis. Hepatology 2005;41(4):779-789.

33. Subramanian A, Sulkowski M, et al. MELD score is an important predictor of pretransplantation mortality in HIV-infected liver transplant candidates. Gastroenterology 2010;138(1):159-164.

34. Muller E, Barday Z, et al. Renal transplantation between HIV-positive donors and recipients justified. S Afr Med J 2012;102(6):497-498.

35. Carithers RL Jr, Herlong HF, et al. Methylprednisolone therapy in patients with severe alcoholic hepatitis. A randomized multicenter trial. Ann Intern Med 1989;110(9):685-690.

36. Maddrey WC, Boitnott JK, et al. Corticosteroid therapy of alcoholic hepatitis. Gastroenterology 1978;75(2):193-199.

37. Louvet A, Naveau S, et al. The Lille model: a new tool for therapeutic strategy in patients with severe alcoholic hepatitis treated with steroids. Hepatology. 2007;45(6):1348-1354.
38. Singal AK, Kamath PS, et al. Alcoholic hepatitis: current challenges and future directions. Clin Gastroenterol Hepatol 2014;12(4):555-564; quiz e531-552.

39. Louvet A, Diaz E, et al. Early switch to pentoxifylline in patients with severe alcoholic hepatitis is inefficient in non-responders to corticosteroids. J Hepatol 2008;48(3):465-470.

40. Dunn W, Jamil LH, et al. MELD accurately predicts mortality in patients with alcoholic hepatitis. Hepatology 2005;41(2):353-358.

41. Lucey MR, Mathurin P, et al. Alcoholic hepatitis. N Engl J Med 2009;360(26):2758-2769.

42. Dew MA, DiMartini AF, et al. Meta-analysis of risk for relapse to substance use after transplantation of the liver or other solid organs. Liver Transpl 2008;14(2):159-172.

43. Mathurin $\mathrm{P}$, Moreno $\mathrm{C}$, et al. Early liver transplantation for severe alcoholic hepatitis. N Engl J Med 2011;365(19):1790-1800.

44. Weeks SR, Sun Z, et al. Liver Transplantation for Severe Alcoholic Hepatitis, Updated Lessons from the World's Largest Series. J Am Coll Surg 2018;226(4):549-557. 